\title{
Lombalgia inespecífica em escolares de 13 a 15 anos
}

Nonspecific low back pain in

schoolchildren aged 13 to 15 years

FisiSenectus. Unochapecó Ano 1, n. 2 - Jul./Dez. 2013 p. 57-64

\section{Jéferson Marchesini}

Bacharel do curso de Graduação em Fisioterapia da Faculdade de Pato Branco.

\section{Solange Maria Bertol Copetti}

Docente do curso de Graduação em Fisioterapia da Faculdade de Pato Branco. Mestre em Educação, Área de Políticas Públicas, na Pontifícia Universidade Católica do Paraná (PUC-PR).

\section{Resumo}

Introdução: a lombalgia inespecífica define-se como dor sem causa identificável, afetando uma parte considerável da população. Essa condição, por sua vez, possui sérias repercussões sobre diferentes níveis (funcional, psicossocial e socioeconômico), sendo a população jovem especial e comumente acometida. Objetivo: o objetivo deste estudo é verificar a existência fator lombalgia inespecífica em escolares com faixa etária de 13 a 15 anos no primeiro semestre letivo de 2010, assim como levantar possíveis causas. Método: foi realizada pesquisa do tipo descritiva e quantitativa. A amostra foi composta por noventa participantes com idade entre de 13 e 15 anos, dos sexos masculino e feminino. 0 material de coleta de dados foi um questionário contendo 16 questões, as quais passaram por um processo de validação. Resultados: de acordo com o questionário, $10 \%$ dos participantes, todos do sexo feminino, tiveram lombalgia inespecífica. 0 fator dor foi apontado em outras regiões do corpo por $53,33 \%$ da amostra e $33,67 \%$ da amostra não tiveram fator dor. Conclusão: a pesquisa apontou um número pequeno de adolescentes com lombalgia inespecífica, correspondendo somente a $10 \%$ dos entrevistados. Foi possível apontar, com base na literatura, prováveis fatores que podem gerar a lombalgia inespecífica.

\section{Palavras-chave}

Fisioterapia. Lombalgia. Saúde escolar.

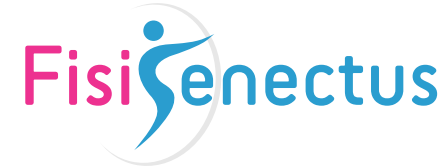




\begin{abstract}
Introduction: the low nonspecific back pain is defined as pain with no identifiable cause that affects a significant proportion of the population. This condition has serious repercussions on different levels (functional, psychosocial and socio-economic). The young persons are the most commonly affected. Objective: the objective of this study is to verify the existence of the nonspecific factor in low back pain in schoolchildren aged 13 to 15 years in the first semester of 2010 as well as raising possible causes. Method: was performed a descriptive research and quantitative. The sample consisted of 90 participants aged between 13 and 15 years, male and female. The data collection was a questionnaire containing 16 questions, which went through a validation process. Results: according to the research $10 \%$ of the participants, all female patients had nonspecific low back pain. The pain factor was pointed out in other parts of the body by $53.33 \%$ of the sample, 33.67\% had no pain factor. Conclusion: the research showed a small number of adolescents with low nonspecific back pain, representing only $10 \%$ of respondents. Based on the literature, it was possible to identify, likely factors that may cause low nonspecific back pain.
\end{abstract}

\title{
Keywords
}

Physical Therapy. Low Back Pain. School Health.

\section{Introdução}

$\infty<\infty<\infty<\infty<\infty<\infty<\infty<\infty<\infty<\infty<\infty<\infty<\infty<\infty<\infty<\infty$

A lombalgia, definida como um sintoma que afeta a região que corresponde às proximidades da coluna lombar e prega glútea, podendo ainda ter irradiação para os membros inferiores, determina um quadro clínico caracterizado por dor e por incapacidade de se movimentar e de trabalhar. Dentre os fatores causais da dor lombar, destacam-se os movimentos de flexão prolongada da coluna, que forçam o núcleo pulposo posteriormente comprimindo o ligamento longitudinal posterior e possivelmente as raízes nervosas, trabalhos com posturas estáticas, tarefas repetitivas, condução prolongada, sedentarismo, fatores psicológicos e pessoais, como idade, sexo, obesidade e posição adotada para dormir. Há também fatores descritos como específicos de causa patológica, tais como hérnia de disco, infecção, inflamações, osteoporose, artrite reumática, fratura ou tumor, e os não específicos, ou seja, sem causa específica ${ }^{1,2}$.

A lombalgia inespecífica define-se como sendo de causa multifatorial que afeta uma parte considerável da população. Essa condição, por sua vez, possui sérias repercussões sobre diferentes níveis (funcional, psicossocial e socioeconômico), sendo a população jovem especial e comumente acometida ${ }^{3,4,5}$.
Durante a idade adulta, acredita-se que ao menos uma vez a pessoa venha a ter ao menos um quadro de dor lombar, fato esse que acomete aproximadamente $70 \%$ a $85 \%$ da população. A Lombalgia inespecífica, que se define como dor sem causa identificável, é responsável por $85 \%$ dos $\operatorname{casos}^{6,7,8,9}$.

Existem alguns fatores que contribuem para um estilo de vida menos ativo dos pré-adolescentes, como disponibilidade de tecnologia, o aumento da insegurança e a progressiva redução dos espaços livres nos centros urbanos (onde vive a maior parte das crianças brasileiras), reduzindo as oportunidades de lazer e de uma vida fisicamente ativa, favorecendo atividades sedentárias, tais como assistir televisão, jogar video games e utilizar computadores ${ }^{10}$.

Dormir e descansar são essenciais para os sistemas muscular, esquelético e nervoso. Durante o sono e o repouso, os músculos relaxam, porém a força da gravidade continua agindo sobre a coluna; e quando esta é suportada por um colchão, altera a forma dos tecidos moles para buscar equilíbrio. Em decúbito lateral, o quadril e o ombro do lado em contato com o colchão tensionam a curva lateralmente, estressando a coluna e seus ligamentos. As pressões intradiscais nos discos lombares nas posições deitada em decúbito dorsal são de $25 \%$ da descarga sobre os discos, enquanto em decúbito lateral são de $75 \%$, quando comparadas à posi- 
ção ortostática, não sendo avaliada a descarga em decúbito ventral ${ }^{11}$.

0 objetivo do presente artigo é verificar a existência fator lombalgia inespecífica em escolares com faixa etária de 13 a 15 anos no primeiro semestre letivo de 2010, assim como levantar possíveis causas.

\section{Materiais e métodos}

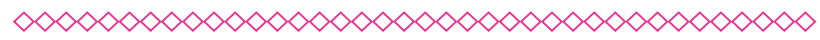

Foi realizada pesquisa do tipo descritiva e quantitativa, na qual "[...] a pesquisa descritiva observa, registra, analisa e correlaciona fatos ou fenômenos (variáveis) sem manipulá-los."12

A amostra foi composta por noventa participantes, com idade entre de 13 e 15 anos, dos sexos masculino e feminino. Destes, 36 participantes tem idade de 13 anos, 43 participantes com 14 anos e 11 participantes com 15 anos.

Os critérios de inclusão na pesquisa foram o fator idade e estarem nas respectivas $7^{\mathrm{a}}$ e $8^{\mathrm{a}}$ séries do ensino fundamental. Como critério de exclusão, usamos os alunos fora da faixa etária da pesquisa pertencente aos grupos pesquisados.

0 material de coleta de dados foi um questionário contendo 16 questões, as quais passaram por um processo de validação. A pesquisa foi realizada no Colégio Estadual Carmela Bortot, na cidade de Pato Branco (PR), no período de 24 a 27 de agosto de 2010. Assim, os questionários foram aplicados com autorização do diretor do colégio, nos quais os alunos não foram identificados, nem foi obtido qualquer tipo de imagem destes. Para aplicar e monitorar os questionários, os professores em horários pré-determinados pela direção fizeram de forma a não interferir no bom funcionamento da instituição no período de quatro dias, sendo os professores orientados a deixar o tempo livre, assim como a livre interpretação, para não influenciar nas respostas dos participantes. As turmas participantes foram duas no período matutino ( $7^{\mathrm{a}}$ e $8^{\mathrm{a}}$ séries) e duas no período vespertino ( $7^{\mathrm{a}}$ e $8^{\mathrm{a}}$ séries). Os dados obtidos nos questionários foram analisados a partir da organização em tabelas e gráficos, em se podem apurar os resultados através do maior percentual obtido nas respostas dadas pelos participantes.

\section{Resultados}

$\infty<\infty<\infty<\infty<\infty<\infty<\infty<\infty<\infty<\infty<\infty<\infty<\infty<\infty<\infty<\infty<\infty<\infty$

Foram entrevistados noventa alunos de 13 a 15 anos, conforme a tabela 1, que se encontra nos anexos. 0 total de participantes na amostra do sexo masculino corresponde a $47,77 \%$ e o total de participantes do sexo feminino corresponde a $52,23 \%$. 0 gráfico 1 , situado nos anexos, corresponde aos participantes que tiveram o fator dor no primeiro semestre letivo, os que apresentaram lombalgia inespecífica e os que não apresentaram o fator dor.

De acordo com o gráfico 1, contendo um total de noventa participantes, nove destes tiveram lombalgia inespecífica, o que corresponde a $10 \%$ do total da amostra, 48 tiveram fator dor em outras regiões do corpo, o que corresponde a 53,33\% da amostra, e 33 não tiveram fator dor, o que corresponde a $33,67 \%$ da amostra.

Os participantes que apresentaram lombalgia inespecífica, que é o objeto de estudo desse artigo, eram todos do sexo feminino, apresentando também dor em outras regiões do corpo, tendo predominância: na cabeça de 50\% (3), membro superior esquerdo $16,66 \%$ (1), membro superior direito $16,66 \%$ (1), membro inferior direito $16,66 \%$ (1). Dor no tronco e membro superior esquerdo não tiveram nenhuma incidência.

Quanto aos participantes que tiveram dor em outras regiões do corpo, sua predominância foi: na cabeça de $14,93 \%$ (10), membro superior esquerdo $11,94 \%$ (8), membro superior direito $14,93 \%$ (10), membro inferior esquerdo $16,41 \%$ (11), membro inferior direito $26,86 \%$ (18), tronco $14,93 \%$ (10), totalizando 67 acometimentos. Desses participantes, oito apresentaram dor em outros segmentos da coluna, sendo $25 \%$ cervicalgia e $75 \%$ dorsalgia.

Seguindo os critérios de inclusão na pesquisa, que é possuir lombalgia inespecífica, utilizamos a escala analógica de dor de Borg para avaliarmos a dor média dos participantes, que atingiu 4,4 pontos. 0 período do dia em que ocorreu maior predominância de dor foi de noite, obtendo 50\% (5), seguida pela tarde, com $30 \%$ (3), e pela manhã $20 \%$ (2). No período do dia que corresponde à madrugada, nenhum participante referiu ter sentido dores. Os participantes foram questionados quan- 
to à procura por profissionais da saúde devido ao quadro álgico e 33,33\% (3) relataram que buscaram ajuda, e os outros $66,67 \%$ (6) relataram que não buscaram ajuda.

Quanto ao meio de transporte utilizado, $55,56 \%$ (5) vão a pé para escola, $11,11 \%$ (1) de carro e $33,33 \%$ (3) de ônibus. Em relação ao tempo utilizado para ir de casa até a escola, $55,56 \%$ (5) levam de 5 a 10 minutos, 22,22\% (2) levam de 10 a 20 minutos, $11,11 \%$ (1) levam de 30 minutos a uma hora e $11,11 \%$ (1) levam mais de uma hora para cumprir o trajeto.

Sobre o tempo destinado a dormir à noite, $11,11 \%$ (1) participantes destinam sete horas, $44,45 \%$ (5) destinam oito horas, $22,22 \%$ (2) destinam nove horas e $22,22 \%$ (2) mais de nove horas. Quanto à qualidade do sono, 22,22\% (2) tem péssima qualidade, $22,22 \%$ (2) regular qualidade de sono, 33,34\% (3) tem boa qualidade e $22,22 \%$ (2) ótima qualidade. A posição decúbito lateral teve 55,56\% de predominância, 33,33\% (3) decúbito ventral e $11,11 \%$ (1) decúbito dorsal. 0 tipo de travesseiro, considerado como alto e baixo pelos participantes, teve o predomínio de $44,44 \%$ (4) para travesseiro baixo, 44,44\% (4) para travesseiro alto e $11,12 \%$ (1) para outro, a qual correspondia à questão aberta e responderam que utilizam travesseiro com espuma do tipo viscoelástica.

De acordo com o gráfico 2, localizado nos anexos, três participantes dedicam-se 1 hora por dia à prática de atividade sedentária, o que corresponde a 33,33\% da amostra, três dedicam-se 2 horas, 0 que corresponde a 33,33\% da amostra, um dedica-se a 3 horas de atividade sedentária, o que corresponde a $11,11 \%$ da amostra, e dois dedicam-se mais de 5 horas, o que corresponde a 22,23\% da amostra. A expressão atividade sedentária refere-se à atividades estáticas, isto é, que não envolvam movimentos com deslocamentos.

Quanto a prática de atividade física regular, $33,33 \%$ (3) responderam que praticam 1 vez na semana, $22,22 \%$ (2) praticam 2 vezes na semana, $22,22 \%$ (2) praticam 3 vezes, $11,11 \%$ (1) praticam 4 vezes ou mais e $11,11 \%$ (1) não praticam atividade física. Quanto ao tempo total destinado à pratica da atividade física, 75\% (6) dedicam-se 1 hora, $12,5 \%$ (1) dedicam-se a 2 horas e 12,5\% (1) mais de
4 horas. A partir do gráfico 3, situado nos anexos, temos que o tipo de mochila mais utilizado é o de costas, que corresponde a $67 \%$ (6), 11\% (1) utilizam mochila de costas, porém, só com uma alça; $11 \%$ (1) utilizam bolsa e $11 \%$ (1) utilizam mochila com alça transversal.

\section{Discussão}

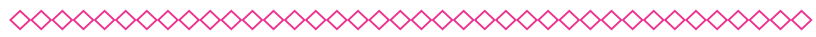

De acordo com os resultados obtidos na pesquisa, 10\% dos participantes tiveram quadro de lombalgia inespecífica, sendo todos do sexo feminino. A dor média, baseada na escala analógica de dor, foi de 4,4 pontos. O período do dia em que ocorreu maior predominância de dor foi à noite, obtendo $50 \%$. Destes, apenas $66,67 \%$ relataram que buscaram ajuda de profissionais da saúde. Estudos revelaram que quanto ao fator sexo existe certa discrepância a respeito do fator lombalgia, pois alguns autores apontam para maior incidência quanto ao sexo feminino ou quanto ao masculino, e outros autores relaram não existir um predomínio entre os $\operatorname{sexos}^{13}$. 0 "período de crescimento acelerado", por existir maior incidência de alterações posturais, segundo alguns autores, pode ser o grande motivo da dor lombar inespecífica ${ }^{14}$. No que diz respeito ao núcleo pulposo, os autores acima discorrem que o núcleo possui certo conteúdo de água que apresenta variações, aumentando seu tamanho no período de repouso. Desta forma, ocorrerá absorção de líquido e, consequentemente, a diminuição de seu tamanho ao longo do dia.

A maioria dos participantes, ou seja, 55,56\%, vai a pé para a escola, e o tempo para cumprir o trajeto é de 5 a 10 minutos. Assim, o tempo utilizado para o deslocamento dos alunos pesquisados não apresenta relevância como fator causal da dor. Quanto ao tempo destinado a dormir de noite, $44,45 \%$ destinam oito horas. Quanto a qualidade do sono, 33,34\% têm boa qualidade. A posição decúbito lateral teve $55,56 \%$ de predominância. 0 tipo de travesseiro considerado como alto e baixo pelos participantes teve predomínio de $44,44 \%$ (4) para travesseiro baixo e $44,44 \%$ (4) para travesseiro alto. 0 decúbito dorsal é a posição mais indicada para relaxamento de queixas de dor na coluna. 
Entretanto, o decúbito ventral poderia ser indicado para uma boa noite de sono se não causasse importantes alterações na coluna cervical. Nesta posição, a porção cervical fica desalinhada com o restante da coluna, permanecendo em rotação associada à hiperextensão da cervical e mantendo a postura inadequada durante as seis a oito horas de sono. Desta forma, indica-se o decúbito lateral as pessoas com queixas de quadro álgico na coluna cervical e lombar, utilizando-se de um travesseiro da altura da cabeça aos ombros. Para evitar compressão do nervo tibial e para alinhar a pelve, também se indica um travesseiro entre os membros inferiores ${ }^{11}$.

De acordo com o gráfico 2, sobre o tempo dedicado a atividades sedentárias, apenas dois participantes dedicam-se mais de 5 horas, o que corresponde a $22,23 \%$ da amostra. Quanto a prática de atividade física regular, 33,33\% (3) responderam que praticam uma vez na semana, 22,22\% (2) praticam duas vezes na semana, $22,22 \%$ (2) praticam três vezes, $11,11 \%$ (1) pratica quatro vezes ou mais e $11,11 \%$ (1) não praticam atividade física. Quanto ao tempo total destinado à pratica da atividade física, 75\% (6) dedicam-se 1 hora, 12,5\% (1) dedicam-se a 2 horas e $12,5 \%$ (1) mais de 4 horas. Existem fatores biomecânicos que podem influenciar na existência da dor, e são divididos em dois grupos: os fatores endógenos, que inclui arquitetura vertebral, que, estando alterada, pode aumentar o risco de forças de stress mecânico em determinadas estruturas das vértebras; e fatores exógenos inerentes a postura adotada, físicos e atividade desportiva ${ }^{14}$. Os estilos de vida incluem essencialmente a prática desportiva intensa ou competitiva e o sedentarismo demasiado, os quais se têm assumido como fatores de grande importância, dada a sua nocividade para os sistemas musculoesquelético e psicológico. Vários autores referem que a prática desportiva muito precoce e/ ou elevadamente intensa podem ter o mesmo risco de desenvolvimento de lombalgias que a ausência de prática física ${ }^{3}$.

0 tipo de mochila mais utilizado é o de costas, que corresponde a $67 \%$ (6); $11 \%$ (1) utilizam mochila de costas, porém, só com uma alça; $11 \%$ (1) utilizam bolsa e $11 \%$ (1) utilizam mochila com alça transversal. Devido à evolução do currículo escolar, aumentam a lista de materiais a serem utilizados. Sabe-se que as crianças irão passar no mínimo oito anos da fase crucial do desenvolvimento transportando seu material escolar. As alterações encontradas para indivíduos que usam mochila de costas foram de três tipos: alterações das curvaturas da coluna, aumento da ação da musculatura lombar e o aumento de compressão intradiscal em $\mathrm{L} 5-\mathrm{S} 1^{14}$.

Se a carga da mochila for superior à capacidade de sustentação dos grupos musculares, ocorrerá sobrecarga para a coluna, podendo determinar alterações na postura. Sendo o transporte do material escolar uma rotina diária, cuidados especiais são necessários para evitar que alterações posturais possam se instalar em médio e longo prazo. Entretanto, autores sugerem que cargas até 10\% da massa corporal possivelmente não ofereçam risco, porém afirmam que mais pesquisas precisam ser feitas. Outros autores sugerem o limite máximo de até $15 \%$ da massa corporal. Apesar disso, tais informações também não são baseadas em dados científicos ${ }^{15}$.

\section{Conclusão}

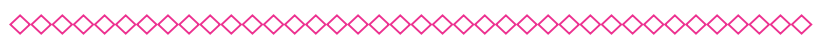

A pesquisa apontou um número pequeno de adolescentes com lombalgia inespecífica, correspondendo somente a $10 \%$ dos entrevistados. Um achado interessante refere-se ao gênero, destacando somente indivíduos do sexo feminino com tal queixa. Os autores pesquisados apontam como fatores causais o crescimento acelerado que ocorre nessa faixa etária, a mochila, a prática desportiva intensa ou competitiva, o sedentarismo demasiado e a posição adotada para dormir. Porém, nesta pesquisa estes dados não foram determinantes no fator lombalgia. Colocamos a questão do uso de salto e a dismenorreia (que não foi pesquisado) como possíveis causas também a serem consideradas, por se tratar de meninas adolescentes na faixa de 13 a 15 anos. Este fato pode apresentar-se como um possível limitador da pesquisa, surgindo, assim, a oportunidade para novas pesquisas abordando esse fator. 


\section{Referências}

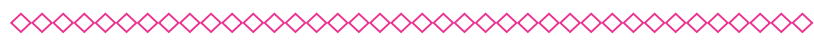

1. Ritter AL, Silva RR. O peso e o transporte do material escolar e a prevalência de dor em estudantes do ensino fundamental [acesso em 02 jul 2012]. Disponível em http://www2.rc.unesp. br/eventos/educacao_fisica/biomecanica2007/ upload/121-2-A-CBB\%20-\%20com\%20autores.pdf

2. Umeta RSG, Santili C, Meves R, Caffaro MFS. Síndromes dolorosas lombares. [acesso em 02 de jul de 2012]. Disponível em http://www.ortopediatrica.com.br/ gtuilojutgtuuooistghiunolsgsstred2aksbgt8 wjsikhol/imprensa/douglas_1022010_85937.pdf

3. Pitangui ACR, Ferreira, CHJ. Avaliação fisioterapêutica e tratamento da lombalgia gestacional. Fisioter. Mov. 2008;21(2):135-142.

4. Goss, CM. Gray Anatomia. 29. ed. Rio de Janeiro: Guanabara Koogan S.A; 1988.

5. Carvalho AR, Gregório FC, Engel GS. Descrição de uma intervenção cinesioterapêutica combinada sobre a capacidade funcional e o nível de incapacidade em portadoras de lombalgia inespecífica crônica. Arq. Ciênc. Saúde UNIPAR, 2009;13(2):97-103.

6. Coelho L, Almeida V, Oliveira R. Lombalgia nos adolescentes: identificação de factores de risco psicossociais. Estudo epidemiológico na Região da Grande Lisboa. Revista Portuguesa De Saúde Pública. 2005;23(1):81-91.

7. Vidal A R. Dor lombar inespecífica em alunos adolescentes em função do gênero, idade e nível de atividade física [Dissertação de Mestrado]. Faculdade de Deporto da Universidade do Porto,2009.
8. Pires RAM, Dumas FLV. Lombalgia: revisão de conceitos e métodos de tratamentos. Universitas: Ciências da Saúde. 2008;6(2):159-168.

9. Pereira NT, Ferreira LAB, Pereira WM. Efetividade de exercícios de estabilização segmentar sobre a dor lombar crônica mecânicopostural. Fisioter. Mov. 2010;23(4):605-614.

10. Carvalho, DA. Os princípios do método Pilates no solo na lombalgia crônica [Monografia]. Tubarão: Universidade do Sul de Santa Catarina; 2006.

11. Pereira FC, Morales JC, Facci L, Palácio SG. Relação da postura adotada para dormir e a queixa de lombalgia. In: Encontro Internacional de Produção Científica Cesumar. Maringá-PR; 2009.

12. Cervo AL, Bervian PA. Metodologia científica. 4. ed. São Paulo: Marron Books Ltda, 1996.

13. Almeida CL, Bermudes JA, Spinelli LHB, Alves MAO, Estevão RA, Santana VA, et al. Morfologia dos discos intervertebrais e abordagem clínica das discopatias: uma revisão bibliográfica. Recife; 2005 [acesso em 02 jul 2012]. Disponível em http://www.wgate.com.br/conteudo/ medicinaesaude/fisioterapia/traumato/ discopatias.htm.

14. Almeida V, Coelho L, Oliveira R. Lombalgia inespecífica nos adolescentes: identificação de factores de risco biomorfológicos. Revista da Essa. 2006;3:65-86.

15. Rodrigues S, Montebelo MIL, Teodori RM. Distribuição da força plantar e oscilação do centro de pressão em relação ao peso e posicionamento do material escolar. Rev Bras Fisioter. 2008;12(1):43-8. 


\section{Anexos}

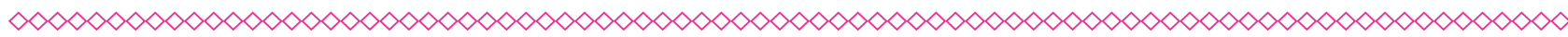

Tabela 1 - Número de participantes por suas respectivas faixas etárias e sexo

\begin{tabular}{ccccc} 
Idade & Masculino & Feminino & Participantes & $\%$ \\
13 & 16 & 20 & 36 & 40 \\
14 & 21 & 22 & 43 & 47,78 \\
15 & 6 & 5 & 11 & 12,22 \\
Total & 43 & 47 & 90 & 100 \\
\hline
\end{tabular}

Fonte: elaboração dos autores.

(clique para voltar ao texto)

Gráfico 1 - Número de participantes e fator dor

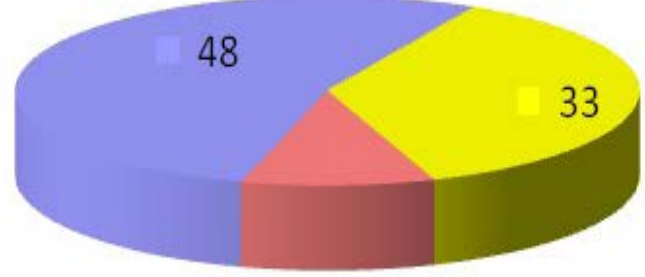

9
- Lombalgia inespecífica

- Dor em outras regiőes

Não tiveram fator dor

(clique para voltar ao texto)

Fonte: elaboração dos autores.

Q1 hora

Q2 horas

प3 horas

$\square 5$ horas ou mais

(clique para voltar ao texto)

Fonte: elaboração dos autores. 
Gráfico 3 - Modelo de objeto utilizado para carregar os materiais escolares

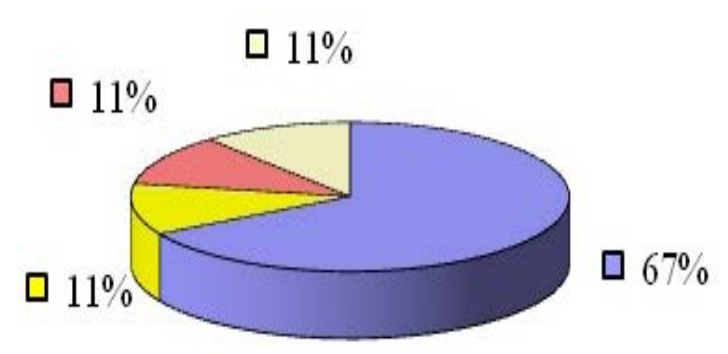

Fonte: elaboração dos autores.
口Mochila de costas

口Mochila de costas, porém, utilizando uma alça

\section{口Bolsa}

(clique para voltar ao texto) 